TRABAJOS ORIGINALES

Rev Obstet Ginecol Venez. 2021; 81 (3): 256-261

https://doi.org/10.51288/00810309

\title{
Caracterización clínica y epidemiológica de pacientes con enfermedad de von Willebrand que consultan por sangrado uterino anormal atendidas en una institución de alto nivel de complejidad
}

\author{
D Lina María Martínez-Sánchez, ${ }^{1}$ (D) Ana María Guevara-Zambrano,, ${ }^{1,2}$ \\ (D) María Patricia Hormaza-Ángel, ${ }^{1}$ (D) Johan Harley Muñoz-Ríos, ${ }^{1}$ (D) Mabel Dahiana Roldán-Tabares, ${ }^{1}$ \\ (D) Laura Isabel Jaramillo-Jaramillo, ${ }^{1}$ (D) Laura Herrera-Almanza, ${ }^{1}$ iD Juan Diego Villegas-Alzate. ${ }^{1}$
}

\begin{abstract}
RESUMEN
Objetivo: Caracterizar clínica y epidemiológicamente las pacientes con enfermedad de von Willebrand y sangrado uterino anormal. Métodos: Estudio observacional descriptivo transversal, en mujeres con diagnóstico de enfermedad de von Willebrand que consultaron en una institución de alta complejidad de la ciudad de Medellín. El análisis se llevó a cabo a través del programa SPSS V 24.

Resultados: Se incluyeron 70 pacientes, 50 de estas fueron descartadas por no presentar hemorragia uterina anormal o tener otros diagnósticos que podian intervenir en la caracterización como síndrome antifosfolípido. La mediana de la edad de las pacientes incluidas fue de 19,5 años (mín: 11, máx: 52), 9 pacientes (60\%) planificaban, los métodos reportados fueron anticonceptivos orales combinados (26,7\%), dispositivos intrauterinos (20\%) y métodos quirúrgicos (13,3\%). En cuanto a la caracterización clínica realizada, se encontró que la presencia de hematomas $40 \%$ (8) y epistaxis $40 \%$ (8) fueron las manifestaciones más frecuentes. La mayoria de las pacientes, $65 \%$ (13) no había recibido tratamiento previo a la consulta para la hemorragia uterina anormal. Respecto a la evaluación de los paraclínicos se encontró: hemoglobina $13 \mathrm{~g} / \mathrm{dL}\left(6,6\right.$ - 15,7), hematocrito 37,5\% (20,3 - 46,1), plaquetas 279000 células $/ \mathrm{mm}^{3}$ (106 000 - 393 000), antígeno plasmático factor de von Willebrand 68,5 UI (19,7-205), actividad plasmática del factor de von Willebrand 44 UI (23 - 126). Conclusión: El sangrado uterino anormal es una manifestación frecuente en mujeres con enfermedad de von Willebrand y puede llegar a presentarse en distintas etapas de la vida, pese a su diagnóstico, las mujeres no reciben manejo de esta manifestación clínica sino hasta su consulta con ginecología.
\end{abstract}

Palabras clave: Enfermedad de von Willebrand, Factor de von Willebrand, Sangrado uterino anormal, Metrorragia.

\section{SUMMARY}

Objective: Characterize clinically and epidemiologically patients with von Willebrand disease and abnormal uterine bleeding. Methods: A cross-sectional descriptive observational study was carried out. The study population was women with a diagnosis of von Willebrand's disease who consulted in a highly complex institution in the city of Medellin. The analysis was carried out through the SPSS V 24 program.

Results: 70 patients were included, 50 of these were discarded because they did not present abnormal uterine bleeding or had other diagnoses that could intervene in the characterization as antiphospholipid syndrome. The median age of the included patients was 19.5 years (Min: 11, Max: 52). 9 patients (60\%) used contraceptive methods, the reported methods were combined oral contraceptives (26.7\%), intrauterine devices (20\%) and surgical methods (13.3\%). Regarding the clinical characterization, it was found that the most frequent manifestations were the presence of bruises $40 \%(8)$ and epistaxis $40 \%$ (8). Most of the patients, $65 \%$ (13) had not received treatment for abnormal uterine bleeding. Laboratory tests showed the following: hemoglobin $13 \mathrm{~g} / \mathrm{dL}$ (6.6 - 15.7), hematocrit 37.5\% (20.3 - 46.1), platelets 279,000 cells $/ \mathrm{mm}^{3}$ (106,000 - 393,000), von Willebrand factor plasma antigen $68.5 \mathrm{IU}(19.7$ - 205), von Willebrand factor plasma activity 44 IU $(23$ - 126).

Conclusion: Abnormal uterine hemorrhage is a frequent manifestation in women with von Willebrand disease and can present at different stages of life, despite its diagnosis, women do not receive management of this clinical manifestation until their consultation with gynecology.

Key words: von Willebrand Diseases, von Willebrand Factor, Abnormal uterine bleeding, Metrorrhagia.

${ }^{1}$ Universidad Pontificia Bolivariana, Calle 78 B N 72 a 109, Medellín, Colombia. Escuela de Ciencias de la Salud, Facultad de Medicina.

${ }^{2}$ Hospital Pablo Tobón Uribe. Calle 78 B N 69 - 240, Medellín, Colombia.
Forma de citar este artículo: Martínez-Sánchez LM, Guevara-Zambrano AM, Hormaza-Ángel MO, Muñoz-Ríos JH, Roldán-Tabares MD, Jaramillo-Jaramillo LI, et al. Caracterización clínica y epidemiológica de pacientes con enfermedad de von Willebrand que consultan por sangrado uterino anormal atendidas en una institución de alto nivel de complejidad. Rev Obstet Ginecol Venez. 2021; 81(3):256-261. https://doi.org/10.51288/00810309 


\section{INTRODUCCIÓN}

La enfermedad de von Willebrand (EVW) es un trastorno hereditario que se caracteriza por hemorragias de variable intensidad y localización, se da por alteraciones cuantitativas o funcionales del factor de von Willebrand (FVW) en el plasma $(1,2)$. Su prevalencia en la población general es de aproximadamente el $1 \%$, siendo mayor en mujeres, aún más en aquellas que presentan sangrado uterino anormal (SUA), con una prevalencia estimada de $13 \%(3-5)$.

Esta es una patología de carácter autosómico, generada por mutaciones en el cromosoma 12, que altera directamente la cantidad o calidad funcional de la proteína. El FVW es una glicoproteína elaborada en los megacariocitos y las células endoteliales, cuya función principal se da en la hemostasia primaria, promoviendo la adhesión plaquetaria y prolongando la vida media del factor VIII (6-8).

El diagnóstico está basado en la sospecha clínica y la alteración de las pruebas de laboratorio que evidencien las alteraciones en el FVW. Se manifiestan clínicamente por hemorragias de localización e intensidad variable, gingivorragia $26 \%$ - $35 \%$, epistaxis $38 \%-63 \%$, sangrado posoperatorio $20 \%-28 \%$, sangrado gastrointestinal $14 \%$, sangrado intraarticular $6 \%$ - $8 \%$, SUA $74 \%$ - $92 \%$, entre otros (9-12). Esta enfermedad hace parte de las causas de SUA, según la clasificación de la Federación Internacional de Ginecología y Obstetricia, pertenece al grupo de coagulopatías, se ha visto que aproximadamente el $13 \%$ de las mujeres que presentan SUA pueden tener una alteración en el FVW $(13,14)$.

Una vez se tenga la sospecha, se deben solicitar exámenes de laboratorio como: hemograma, FVW, cofactor de ristocetina, tiempos de coagulación, factor VIII, capacidad de unión al factor VIII y unión del FVW al colágeno. El patrón paraclínico más común de presentación es la prolongación de los tiempos de coagulación, disminución de la concentración y actividad del FVW y disminución de la acción del cofactor de ritocetina $(1,15)$.

Para el tratamiento se han descrito dos tipos de terapia: los coadyuvantes de la coagulación que permiten un proceso hemostático indirecto como el ácido tranexámico y el ácido épsilon aminocaproico; y los promotores de la liberación del FVW, como la desmopresina, que aumentan la concentración de este en sangre, por lo tanto, impacta de manera directa en la hemostasia $(6,15)$. Con relación al tratamiento del SUA y la EVW, se ha evidenciado que el uso de dispositivo intrauterino liberador de levonorgestrel puede ser efectivo, igualmente el uso de anticonceptivos orales combinados, sin embargo, los estudios son limitados $(9,16,17)$.

El objetivo del presente estudio fue caracterizar clínica y epidemiológicamente las pacientes con enfermedad de von Willebrand y sangrado uterino anormal.

\section{MÉTODOS}

Diseño y población de estudio. Se realizó una serie de casos, que incluyó las historias clínicas de todas las pacientes con EVW que fueron atendidas en una institución de alto nivel de complejidad durante los años 2015 a 2018, que cumplían con los criterios de elegibilidad: mujeres de todas las edades con diagnóstico de EVW. Los criterios de exclusión utilizados fueron aquellas que presentaban sangrado posmenopáusico, neoplasias y embarazadas. Se realizó un muestreo no probabilístico de casos consecutivos.

Técnica e instrumentos de recolección. La información necesaria para el estudio se recolectó a partir de la revisión cuidadosa de historias clínicas que se encontraban en el archivo interno de la institución de alto nivel de complejidad. El proceso de recolección 
de datos fue realizado por los investigadores a través de un formulario previamente diseñado que incluía todas las variables de estudio.

Procesamiento de la información y plan de análisis. La información recolectada fue digitada en una base de datos, las variables allí ingresadas se validaron con el fin de disminuir los posibles sesgos. Se realizó una revisión periódica de los registros que permitió verificar la calidad de la información obtenida. El análisis se llevó a cabo a través del programa SPSS, versión 24, empleando un análisis univariado; para las variables cualitativas se usó frecuencias absolutas y relativas, para las cuantitativas promedio y desviación estándar o mediana e índices intercuartílicos, según corresponda de acuerdo con su distribución.

Consideraciones éticas. La investigación contó con la aprobación ética de la institución donde se llevó a cabo y según la resolución 008430 de 1993 del Ministerio de Salud de Colombia fue clasificado como una investigación sin riesgo.

\section{RESULTADOS}

Se revisaron 70 pacientes, 50 de estas fueron descartadas por no presentar hemorragia uterina anormal o tener otros diagnósticos que podían intervenir en la caracterización como síndrome antifosfolípido por lo que la muestra final fue de 20 pacientes.

El rango de edad de las pacientes estuvo entre los $11 \mathrm{y}$ 52 años, con una mediana de 19,5 años. Solo se obtuvo la información de 8 pacientes respecto a la edad de la menarquia, con un promedio de 11,5 años.

Evaluando los antecedentes personales, 1 (5 \%) de las pacientes tenía dentro de sus antecedentes personales hipotiroidismo y era manejada con $50 \mathrm{mcg}$ de levotiroxina. Respecto a la historia obstétrica de la población estudiada, se encontró que todas las pacientes eran nuligestantes, de acuerdo a lo reportado en las historias clínicas. En cuanto a los antecedentes ginecológicos, no había información completa reportada respecto a la citología, solo 1 (5 $\%)$ paciente tenía registrado un resultado negativo. Al evaluar cuáles eran los métodos de planificación familiar, $9(60 \%)$ pacientes hacían uso de estos, los métodos reportados fueron anticonceptivos orales combinados, 4 (26,7 \%) pacientes, dispositivos intrauterinos, 3 (20\%) y métodos quirúrgicos, 2 (13,3\%), una de las pacientes además de anticonceptivos orales combinados también había empleado dispositivo intrauterino; del resto de las pacientes no se especificaba el método de planificación.

Respecto al ciclo menstrual, se encontró reportado en las historias clínicas información sobre el volumen de sangrado en 4 (20\%) pacientes. Los otros datos se detallan en la tabla 1.

En cuanto a la caracterización clínica realizada, se encontró que la presencia de hematomas y epistaxis fueron las manifestaciones más frecuentes; en la tabla 2 se pueden observar con mayor detalle los hallazgos. Adicionalmente, se reportó el sangrado

Tabla 1. Características del ciclo menstrual

\begin{tabular}{lcc}
\hline Característica & \multicolumn{2}{c}{ Medida } \\
\hline & Irregular & $8(40 \%)$ \\
Regularidad & Regular & $6(30 \%)$ \\
& No hay datos & $6(30 \%)$ \\
Periodicidad & Promedio 29,6 días \\
Duración del sangrado & Mediana 7,5 días \\
Número de toallas & Mediana 4 toallas \\
Tiempo de evolución del & Min 3/Max 10 \\
sangrado uterino anormal & Mediana 18 meses \\
\hline
\end{tabular}


Tabla 2. Manifestaciones clínicas

\begin{tabular}{ll}
\hline Síntoma & $\mathrm{n}(\%)$ \\
\hline Hematomas & $8(40)$ \\
Epistaxis & $8(40)$ \\
Gingivorragia & $6(30)$ \\
Petequias & $3(15)$ \\
Dismenorrea & $3(15)$ \\
\hline
\end{tabular}

menstrual prolongado, el sangrado intermenstrual y las hemorragias posquirúrgicas como otras manifestaciones adicionales.

El $65 \%$ (13) de las pacientes no había recibido tratamiento previo a la consulta para el SUA, el $25 \%$ (5) había recibido anticonceptivos orales combinados, el $10 \%$ (2) había recibido anticonceptivos orales de solo progestágenos y empleado implantes intrauterinos respectivamente y el $5 \%$ (1) recibió ácido tranexámico. Estos porcentajes fueron calculados sobre el $\mathrm{n}$ de 20 pacientes, pero una misma paciente había podido recibir varios tratamientos ya que no son excluyentes.

En la tabla 3 se detallan los valores de laboratorio de las pacientes estudiadas. Respecto a la prolactina, solo se encontró reportado en la historia clínica de una paciente con valor de $17,32 \mathrm{ng} / \mathrm{mL}$.

Solo $5(25 \%)$ pacientes tenían información respecto a la valoración ultrasonográfica de la anatomía de los órganos pélvicos, en 3 de estas se hallaron quistes simples anexiales, una tenia endometrio engrosado y otra miomatosis uterina.

Los tratamientos definidos para las pacientes, después de la evaluación, se presentan en la tabla 4. En ninguna de las historias clínicas se encontró reportada la realización de legrado.
Tabla 3. Distribución de tumores, de acuerdo a su localización en la mama

\begin{tabular}{lc}
\hline Laboratorio & $\begin{array}{c}\text { Mediana } \\
\text { (Menor - Mayor) }\end{array}$ \\
\hline Hemoglobina g/dL & $13(6,6-15,7)$ \\
Hematocrito \% & $37,5(20,3-46,1)$ \\
VCM fL & $87,96(64,7-94)$ \\
HCM pg & $27,1(24-30,2)$ \\
ADE \% & $14,8(12,8-16,9)$ \\
Leucocitos $10^{\wedge} 3 / \mu \mathrm{L}$ & $7,46(3,90-10,00)$ \\
Recuento neutrófilos $10^{\wedge} 3 / \mu \mathrm{L}$ & $4,04(1,90-7,60)$ \\
Recuento de linfocitos 10^3/ $\mu \mathrm{L}$ & $1,87(1,62-4,12)$ \\
Recuento de plaquetas $10^{\wedge} 3 / \mu \mathrm{L}$ & $2,79(1,06-3,93)$ \\
Tiempo de protrombina segundos & $13,1(10-26,3)$ \\
Tiempo de tromboplastina segundos & $30,45(9,9-46)$ \\
Fibrinógeno mg/dL & $392(254-459)$ \\
Antígeno plasmático FVW UI/dL & $68,5(19,7-205)$ \\
Actividad plasmática FVW \% & $44(23-126)$ \\
Actividad factor VIII \% & $66(36-116)$ \\
TSH mIU/L & $1,57(0,64-13)$ \\
\hline
\end{tabular}

Tabla 4. Tratamientos definidos

\begin{tabular}{lc}
\hline Tratamiento & $\mathrm{n}(\%)$ \\
\hline Antiinflamatorios no esteroideos & $1(5)$ \\
Ácido tranexámico & $13(65)$ \\
Histerectomía & $3(15)$ \\
Dispositivo intrauterino & $1(5)$ \\
Anticonceptivos orales & $10(50)$ \\
Hierro & $6(30)$ \\
Transfusión & $1(5)$ \\
Factor VIII & $3(15)$ \\
Desmopresina & $2(10)$ \\
\hline
\end{tabular}




\section{DISCUSIÓN}

Los SUA puberales se producen como manifestación inicial de una coagulopatía aproximadamente en un $20 \%$, la EVW representa entre el $11 \%$ y el $20 \%$ de estas (1).

En el estudio realizado en Taiwán por Chen y cols. (18), en mujeres con diagnóstico de EVW, reportaron una mediana de edad de 34,3 años con las siguientes medias para los datos del hemoleucograma: hemoglobina $8,67 \mathrm{~g} / \mathrm{dL}$, hematocrito $28,31 \%$, VCM 64,21 fL y HCM 19,67 pg; datos muy similares a los reportados por Kushwaha y cols. (19), con una media de hemoglobina de $6,9 \mathrm{~g} / \mathrm{dL}$ y VCM de $68 \mathrm{fL}$. En el presente estudio, la mediana de edad fue de 19,5 años $\mathrm{y}$ todos los valores de laboratorio mencionados fueron superiores.

Respecto al antígeno plasmático del FVW, la mediana del presente estudio fue de $68,5 \mathrm{UI} / \mathrm{dL}$, dato superior al reportado por Kushwaha y cols. (19) con un promedio de $48,8 \mathrm{UI} / \mathrm{dL}$, al igual que el dato reportado por Lavin y cols. (20) de $59 \mathrm{UI} / \mathrm{dL}$.

Kadir y cols. (21), en Reino Unido, evaluaron pacientes que tenían diagnóstico de $\mathrm{EVW}$, y reportaron una media de edad de 40 años y para la duración del sangrado menstrual de 6,5 días, este último dato es similar en la presente investigación. En el mismo estudio, reportaron la necesidad de transfusión en $3(15 \%)$ pacientes mientras en el presente estudio se reportó necesidad de transfusión solo en $1(5 \%)$ paciente.

En cuanto a los síntomas presentados por las pacientes del estudio de Kadir y cols. (21), los principales fueron hematomas, gingivorragia y epistaxis con $16(80 \%), 9$ ( $45 \%)$ y $5(25 \%)$ respectivamente; datos comparables con el presente estudio en el que estos síntomas fueron los predominantes.
Con relación al tratamiento, en el estudio realizado por Lavin y cols. (20) en mujeres con disminución del FVW, utilizaron coadyuvantes de la coagulación (antifibrinolíticos) en $23(19,2 \%)$ de las pacientes y promotores de la coagulación, en este caso la desmopresina, en $1(0,8 \%)$ paciente. En la presente investigación se utilizó en $13(65 \%)$ y 2 (10\%), respectivamente. Así mismo, en el estudio descrito, para el tratamiento del SUA se evidenció la utilización de terapia hormonal en $47(39,2 \%)$ pacientes, dispositivos intrauterinos en $20(16,7 \%)$ pacientes e histerectomía en $9(9,5 \%)$ pacientes, datos comparables con los reportados en el presente estudio.

Se concluye que en el estudio del SUA, la EVW se debe tener en cuenta como sospecha diagnóstica asociada a los antecedentes e historia clínica, pues es una manifestación frecuente de esta enfermedad; se puede desarrollar a cualquier edad, especialmente durante la pubertad. Con relación al tratamiento, se ha evidenciado que no hay un manejo dirigido hacia el SUA hasta la consulta con ginecología, lo cual es un punto importante pues esta manifestación repercute en la salud, entendida como el estado de bienestar total, en las pacientes.

Dentro de las limitaciones de este estudio se incluyen el diseño retrospectivo y descriptivo del estudio, la confianza en el adecuado diligenciamiento de las historias clínicas y la disponibilidad precisa de los datos.

\section{REFERENCIAS}

1. Espitia-De La Hoz F. Manejo de la enfermedad de von Willebrand en ginecología y obstetricia. Revisión cualitativa de la literatura. Rev Colomb Obstet Ginecol. 2010; 61(2): 151-159. doi: https://doi.org/10.18597/ $\operatorname{rcog} .284$

2. Nilsson IM. In memory of Erik Jorpes. von Willebrand's disease from 1926-1983. Scand J Haematol Suppl [Internet]. 1984 [consultado: 10 de agosto de 2020]; 40:21-43. Disponible en: https://onlinelibrary.wiley. com/doi/epdf/10.1111/j.1600-0609.1984.tb02543.x 
3. Seravalli V, Linari S, Peruzzi E, Dei M, Paladino E, Bruni V. Prevalence of hemostatic disorders in adolescents with abnormal uterine bleeding. J Pediatr Adolesc Gynecol. 2013; 26(5):285-289. doi: 10.1016/j. jpag.2013.06.003.

4. Mikhail S, Kouides P. von Willebrand disease in the pediatric and adolescent population. J Pediatr Adolesc Gynecol. 2010; 23(6 Suppl):S3-10. doi: 10.1016/j. jpag.2010.08.005.

5. Vo KT, Grooms L, Klima J, Holland-Hall C, O’Brien $\mathrm{SH}$. Menstrual bleeding patterns and prevalence of bleeding disorders in a multidisciplinary adolescent haematology clinic. Haemophilia. 2013; 19(1):71-75. doi: 10.1111/hae.12012.

6. Toro Cubides AM, Aguilar Posada AS. Enfermedad de Von Willebrand tipo III en una paciente obstétrica. Univ Med. 2019; 60(4):66-74. https://doi.org/10.11144/ javeriana.umed60-4.vonw.

7. Werner EJ, Broxson EH, Tucker EL, Giroux DS, Shults J, Abshire TC. Prevalence of von Willebrand disease in children: a multiethnic study. J Pediatr. 1993; 123(6):893-898. doi: 10.1016/s0022-3476(05)80384-1.

8. Hassan MI, Saxena A, Ahmad F. Structure and function of von Willebrand factor. Blood Coagul Fibrinolysis. 2012; 23(1):11-22. doi: 10.1097/ MBC.0b013e32834cb35d.

9. Committee on Adolescent Health Care; Committee on Gynecologic Practice. Committee Opinion No.580: von Willebrand disease in women. Obstet Gynecol. 2013; 122(6):1368-1373. doi: 10.1097/01. AOG.0000438961.38979.19

10. James AH, Kouides PA, Abdul-Kadir R, Dietrich $\mathrm{JE}$, Edlund $\mathrm{M}$, Federici $\mathrm{AB}$, et al. Evaluation and management of acute menorrhagia in women with and without underlying bleeding disorders: consensus from an international expert panel. Eur J Obstet Gynecol Reprod Biol. 2011; 158(2):124-134. doi: 10.1016/j. ejogrb.2011.04.025.

11. Valente MJ, Abramson N. Easy bruisability. South Med J. 2006; 99(4):366-370. doi: 10.1097/01. smj.0000209237.73616.33.

12. Nichols WL, editor. The diagnosis, evaluation, and management of von Willebrand disease. NIH Publication No. 08-5832 [Internet]. Bethesda (MD): National Heart, Lung, and Blood Institute; 2007 [consultado: 10 de agosto de 2020]. Disponible en: https://www.nhlbi.nih.gov/files/docs/guidelines/vwd. pdf
13. Munro MG. Practical aspects of the two FIGO systems for management of abnormal uterine bleeding in the reproductive years. Best Pract Res Clin Obstet Gynaecol. 2017; 40:3-22. doi: 10.1016/j.bpobgyn.2016.09.011.

14. Shankar M, Lee CA, Sabin CA, Economides DL, Kadir RA. von Willebrand disease in women with menorrhagia: a systematic review. BJOG. 2004; 111(7):734-740. doi: 10.1111/j.1471-0528.2004.00176.x.

15. Sadler JE. A revised classification of von Willebrand disease. For the Subcommittee on von Willebrand Factor of the Scientific and Standardization Committee of the International Society on Thrombosis and Haemostasis. Thromb Haemost [Internet]. 1994 [consultado: 10 de agosto de 2020]; 71(4):520-525. Disponible en: https:// pubmed.ncbi.nlm.nih.gov/8052974/

16. Kingman CE, Kadir RA, Lee CA, Economides DL. The use of levonorgestrel-releasing intrauterine system for treatment of menorrhagia in women with inherited bleeding disorders. BJOG. 2004; 111(12):1425-1428. doi: 10.1111/j.1471-0528.2004.00305.x.

17. Kadir RA, Lukes AS, Kouides PA, Fernandez H, Goudemand J. Management of excessive menstrual bleeding in women with hemostatic disorders. Fertil Steril. 2005; 84(5):1352-1359. doi: 10.1016/j. fertnstert.2005.04.062.

18. Chen YC, Chao TY, Cheng SN, Hu SH, Liu JY. Prevalence of von Willebrand disease in women with iron deficiency anaemia and menorrhagia in Taiwan. Haemophilia. 2008; 14(4):768-774. doi: 10.1111/j.1365-2516.2008.01777.x.

19. Kushwaha R, KumarA, Mishra KL, Sankhwar PL, Singh R. Haemostatic Disorder in Women with Unexplained Menorrhagia: A Tertiary Care Centre Experience from Northern India. J Clin Diagn Res. 2017; 11(5):EC46EC49. doi: 10.7860/JCDR/2017/25722.9928.

20. Lavin M, Aguila S, Dalton N, Nolan M, Byrne M, Ryan $\mathrm{K}$, et al. Significant gynecological bleeding in women with low von Willebrand factor levels. Blood Adv. 2018; 2(14):1784-1791. doi: 10.1182/ bloodadvances.2018017418.

21. Kadir RA, Economides DL, Sabin CA, Owens D, Lee CA. Frequency of inherited bleeding disorders in women with menorrhagia. Lancet. 1998; 351(9101):485-489. doi: 10.1016/S0140-6736(97)08248-2.

Recibido 8 de octubre de 2020 Aprobado 15 de enero de 2021 